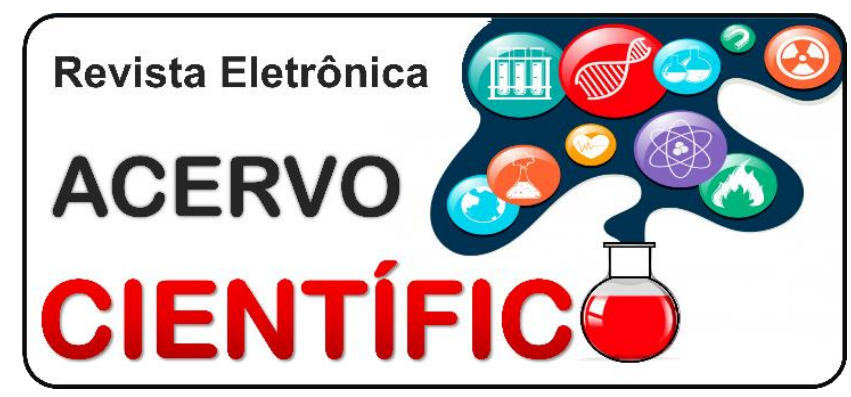

ARTIGO ORIGINAL

Recebido em: 8/2020

Aceito em: 9/2020

Publicado em: 12/2020

\title{
Dinâmica do exame preventivo de câncer de colo de útero em uma unidade de Belém-Pará
}

\author{
Dynamics of cervical cancer preventive examination in a unit in Belém-Pará
}

Dinámica del examen preventivo de câncer cervical en una unidad em Belém-Pará

Lucian Herlan da Costa Luz Fernandes ${ }^{1 *}$, Rodrigo Lagares da Silva ${ }^{1}$, Alicia Renata Sales da Conceição ${ }^{1}$, Rita de Cássia Barroso ${ }^{1}$, Vitória Conceição Batista ${ }^{1}$, José Antonio Cordero da Silva ${ }^{1}$, Mariane Cordeiro Alves Franco'.

Resumo: Descrever a dinâmica da realização do exame preventivo do câncer do colo do útero na Unidade Básica de Saúde em uma cidade do Pará, no período de outubro de 2018 a janeiro de 2019. Trata-se de uma pesquisa observacional do tipo transversal, por meio de um questionário assinado por mulheres atendidas na Unidade Básica de Saúde escolhida. O projeto foi aprovado no Comitê de Ética sob CAAE: 99998918.8.0000.5701. O perfil socioeconômico das mulheres que 18,4\% não costumam realizar o exame periodicamente, idade média de 41,8 anos, sendo a viuvez, baixa nível de escolaridade e renda econômica mensal como fatores associados a não realizar o exame, bem como desconhecimento sobre objetivos do exame, não possuir atividade sexual e utilizar a tabelinha como método contraceptivo. Discussão: Ressaltase que as informações coletadas na pesquisa corroboram a literatura de diversas regiões do país, prejudicando o diagnóstico precoce de patologias (neoplásicas e infecciosas pela inspeção do canal cervical e colo) e qualidade de vida das mulheres. Logo, foi fundamental desenvolver a pesquisa para reconhecer o perfil das mulheres que não realizam o exame a fim de melhorar a educação em saúde, sendo uma metodologia que deve ser utilizada em outras áreas do país.

Palavras-chave: Serviços preventivos de saúde, Neoplasias, Atenção primária à saúde.

Abstract: Describe the dynamics of the cervical cancer preventive exam in the Basic Health Unit on a city of Pará, from October 2018 to January 2019. This is an observational cross-sectional research, using a questionnaire signed by women attended at the chosen Basic Health Unit. The project was approved by the Ethics Committee under CAAE: 99998918.8.0000.5701. The socioeconomic profile of women, $18.4 \%$ of whom do not regularly undergo the exam, average age of 41.8 years, with widowhood, low level of education and monthly economic income as factors associated with not having the exam, as well as ignorance about exam objectives, not having sexual activity and using the tablet as a contraceptive method. Discussion: It is noteworthy that the information collected in the research corroborates the literature from different regions of the country, impairing the early diagnosis of pathologies (neoplastic and infectious through inspection of the cervical canal and cervix) and women's quality of life. Therefore, it was essential to develop the research to recognize the profile of women who do not take the exam in order to improve health education, being a methodology that should be used in other areas of the country.

Keywords: Preventive health services, Neoplasms, Primary health care.

Resumen: Describa la dinámica del examen preventivo del cáncer de cuello uterino en la Unidad Básica de Salud en una cidad del Pará, de octubre de 2018 a enero de 2019. Esta es una investigación observacional de corte transversal, utilizando un cuestionario firmado por mujeres atendidas en la Unidad Básica de Salud

${ }^{1}$ Centro Universitário Metropolitano da Amazônia (UNIFAMAZ), Belém - PA. *E-mail: lucianfernandes95@gmail.com 
elegida. El proyecto fue aprobado por el Comité de Ética bajo CAAE: 99998918.8.0000.5701. El perfil socioeconómico de las mujeres, el $18.4 \%$ de las cuales no se someten regularmente al examen, la edad promedio de 41.8 años, con viudez, bajo nivel de educación e ingresos económicos mensuales como factores asociados con la no realización del examen, así como la ignorancia sobre objetivos del examen, no tener actividad sexual y usar la tableta como método anticonceptivo. Discusión: Cabe destacar que la información recopilada en la investigación corrobora la literatura de varias regiones del país, lo que perjudica el diagnóstico precoz de patologías (neoplásicas e infecciosas mediante inspección del canal cervical y el cuello uterino) y la calidad de vida de las mujeres. Por lo tanto, era esencial desarrollar la investigación para reconocer el perfil de las mujeres que no toman el examen con el fin de mejorar la educación para la salud, siendo una metodología que debería utilizarse en otras áreas del país.

Palabras clave: Servicios preventivos de salud, Neoplasias, Atención primaria de salud.

\section{INTRODUÇÃO}

Um dos tipos de câncer mais recorrentes no mundo é o câncer de colo do útero (SILVA MRF, et al., 2016). Entre os canceres que acometem as mulheres, esta neoplasia só é superada pelos canceres de pele e de mama (BARCELO MRB, et al., 2014). Em nível mundial causa, aproximadamente, seis bilhões de óbitos por ano (MELO MCS, et al., 2012). Na maioria dos casos, a evolução do câncer do colo do útero ocorre de forma lenta, passando por fases pré-clínicas detectáveis e curáveis. Ante a esse quadro, sua prevenção e seu tratamento inserem-se no âmbito da saúde da mulher, área considerada estratégica para ações prioritárias no Sistema Único de Saúde (SUS), no nível da atuação primária. A criação do Programa de Saúde da Família (PSF), em 1994, renomeado como Estratégia Saúde da Família (ESF) em 1996, foi imprescindível para o alargamento da prevenção desse câncer em todo o território nacional (SOUZA AF, et al., 2015).

Destaca-se como relevante nessa atividade a realização do exame preventivo (Papanicolau ou citopatológico), capaz de detectar as lesões precursoras dessa neoplasia. Quando essas alterações antecedentes ao câncer são diagnosticadas e tratadas é possível prevenir totalmente os casos (FACCHINI LA, et al., 2015; AMARAL MS, 2017). Estudos revelam que dificuldades econômicas, geográficas, insuficiência de serviços, questões culturais, como medo, desconsideração de sintomas importantes e preconceito, podem estar associados à não realização de exames preventivos e à incidência dessa doença (DAMACENA AM, 2017). Outras pesquisas apontam a possibilidade desse carcinoma estar relacionado aos seguintes fatores: múltiplos parceiros sexuais, tabagismo, início precoce da atividade sexual, uso de contraceptivos orais por longo tempo, entre outros. Desse modo, é fundamental atentar-se para os motivos que podem interferir na decisão da mulher em realizar o exame preventivo do câncer do colo do útero, pois esses podem estar ligados a tabus, valores culturais e sua própria sexualidade (SOUZA GG, 2011).

O objetivo da presente pesquisa é descrever a dinâmica da realização do exame preventivo do câncer do colo do útero na Unidade Básica de Saúde escolhida para a pesquisa, no período de outubro de 2018 a janeiro de 2019.

\section{MÉTODOS}

O presente estudo foi desenvolvido na forma observacional, do tipo transversal, de caráter descritivo. A pesquisa foi realizada em uma Unidade Básica de Saúde localizada em uma cidade do Pará, referência em saúde da família. As mulheres que participaram dessa pesquisa foram avaliadas de acordo com as determinações da Resolução № 466/2012 do Conselho Nacional de Saúde (CNS), sendo a pesquisa avaliada e autorizada pelo Comitê de Ética em Pesquisa sob número de parecer 3.165.824. O referido estudo teve como população amostral todas as mulheres atendidas de outubro de 2018 a janeiro de 2019. O mecanismo de coleta dos dados foi realizado por meio de protocolo próprio de pesquisa com perguntas abertas e fechadas. Os dados referentes às características socioeconômicas e epidemiológicas foram tratados utilizando estatística descritiva, expressos sob a forma de média \pm desvio padrão, intervalo de Confiança de $95 \%$, mediana e percentis e/ou de frequências absoluta e relativa, conforme o caso, e apresentados em tabelas e/ou gráficos utilizando o Teste G e Teste de Mann-Whitney por meio do programa Bioestat versão 5.3 . 


\section{RESULTADOS}

A Tabela 1 exibe as características demográficas das usuárias incluídas no estudo. Nas colunas estão representadas as usuárias que realizam o exame periodicamente (coluna "Realiza") ou que não realizam o exame periodicamente (coluna "Não Realiza"). Observa-se que $58(18,4 \%)$ mulheres não costumam realizar o exame periodicamente, que a idade média geral é de 41,8 anos, $41,6 \%$ das mulheres é solteira, $51,1 \%$ possui ocupação "Do lar", $57,1 \%$ possui apenas o nível médio, $51,1 \%$ reside com até três pessoas, em residência de alvenaria $(83,7 \%)$ e com renda média de um a dois salários mensais. Também, a idade, o estado civil, a ocupação, o número de pessoas com as quais reside e o tipo de moradia não diferiram significativamente entre os dois grupos.

Por outro lado, houve um número significativamente maior de viúvas entre as que não realizam periodicamente o exame. Quanto à escolaridade, observou-se um maior número de mulheres com apenas o nível fundamental ou analfabeta que não realiza o exame, mais do que se esperaria ao acaso, sugerindo que a decisão de não realizar o exame sofre influência, também, do nível de escolaridade das usuárias. Quanto à renda observou-se que o número de pessoas que não se submetem regularmente ao exame é maior entre quem recebe menos que um salário, enquanto que as que realizam o exame e recebem mais de dois salários foi significativamente maior do que seria esperado ao acaso.

Tabela 1 - Características demográficas das usuárias do serviço de prevenção do câncer de colo uterino da Unidade Básica de Saúde escolhida, avaliadas no período de outubro de 2018 a janeiro de 2019, em uma cidade do Pará.

\begin{tabular}{|c|c|c|c|c|}
\hline Variável & Geral & $\begin{array}{l}\text { Realiza } \\
(\mathrm{n}=257)\end{array}$ & $\begin{array}{c}\text { Não Realiza } \\
(n=58)\end{array}$ & p-valor \\
\hline \multicolumn{5}{|l|}{ Idade (anos) } \\
\hline Mínimo & 17 & 17 & 18 & \multirow{4}{*}{$0,436^{1}$} \\
\hline Máximo & 84 & 80 & 84 & \\
\hline Mediana & 38 & 38,5 & 36 & \\
\hline Média \pm DP & $41,8 \pm 17$ & $41 \pm 15,7$ & $45,2 \pm 21,8$ & \\
\hline \multicolumn{5}{|l|}{ Estado civil } \\
\hline Solteiro & $128(41,6)$ & $104(41,3)$ & $24(42,9)$ & \multirow{4}{*}{$0,010^{2}$} \\
\hline Casado & $94(30,5)$ & $83(32,9)$ & $11(19,6)$ & \\
\hline Outros & $53(17,2)$ & $45(17,9)$ & $8(14,3)$ & \\
\hline Viúvo & $33(10,7)$ & $20(7,9)^{*}$ & $13(23,2) \dagger$ & \\
\hline \multicolumn{5}{|l|}{ Ocupação } \\
\hline Do lar & $157(51,1)$ & $124(49,6)$ & $33(57,9)$ & \multirow{3}{*}{$0,113^{2}$} \\
\hline Assalariado & $93(30,3)$ & $82(32,8)$ & $11(19,3)$ & \\
\hline Trabalhador Autônomo & $57(18,6)$ & $44(17,6)$ & $13(22,8)$ & \\
\hline \multicolumn{5}{|l|}{ Escolaridade } \\
\hline Médio & $180(57,1)$ & $157(61,1) \dagger$ & $23(39,7)^{*}$ & \multirow{4}{*}{$<0,001^{2}$} \\
\hline Fundamental & $70(22,2)$ & $50(19,5)^{*}$ & $20(34,5) \dagger$ & \\
\hline Superior $^{3}$ & $49(15,6)$ & $43(16,7)$ & $6(10,3)$ & \\
\hline Analfabeto & $16(5,1)$ & $7(2,7)^{*}$ & $9(15,5) \dagger$ & \\
\hline \multicolumn{5}{|c|}{ Reside com quantas pessoas } \\
\hline Três ou menos & $160(51,1)$ & $135(52,9)$ & $25(43,1)$ & \multirow{2}{*}{$0,227^{2}$} \\
\hline Mais de três & $153(48,9)$ & $120(47,1)$ & $33(56,9)$ & \\
\hline \multicolumn{5}{|l|}{ Tipo de moradia } \\
\hline Alvenaria & $261(83,7)$ & $218(85,8)$ & $43(74,1)$ & \multirow{2}{*}{$0,059^{2}$} \\
\hline Madeira, palafita, outros & $51(16,3)$ & $36(14,2)$ & $15(25,9)$ & \\
\hline \multicolumn{5}{|l|}{ Renda } \\
\hline Menos de um salário & $45(15,4)$ & $28(11,8)^{*}$ & $17(30,9) \dagger$ & \multirow{3}{*}{$<0,001^{2}$} \\
\hline Um a dois salários & $214(73,3)$ & $178(75,1)$ & $36(65,5)$ & \\
\hline Mais de dois salários & $33(11,3)$ & $31(13,1) \dagger$ & $2(3,6)^{*}$ & \\
\hline
\end{tabular}

Legenda: As variáveis categóricas são representadas como n (\%). DP: Desvio Padrão. ${ }^{1}$ : Teste U de Mann-Whitney. 2: Teste G. ${ }^{3}$ : Ensino superior completo ou incompleto. *: A frequência observada foi inferior ao que seria esperado ao acaso. $\dagger$ : A frequência observada foi superior ao que seria esperado ao acaso.

Fonte: Fernandes LHCL, et al., 2020. 
A Tabela 2 mostra que houve associação significativa entre a decisão de realizar periodicamente o exame preventivo e a sexarca, a frequência sexual e o uso de métodos contraceptivos. A decisão de não realizar periodicamente é mais frequente em quem teve a sexarca após os 18 anos e entre quem não usa métodos contraceptivos. Também, quem não relata nenhuma atividade sexual semanal é mais propensa a não realizar os exames, enquanto quem possui mais de três relações está mais propensa a realizar os exames periodicamente.

Tabela 2 - Características sexuais das usuárias do serviço de prevenção do câncer de colo uterino da Unidade Básica de Saúde escolhida, avaliadas no período de outubro de 2018 a janeiro de 2019, em uma cidade do Pará.

\begin{tabular}{lcccc}
\hline Variável & Geral & $\begin{array}{c}\text { Realiza } \\
(\mathbf{n}=\mathbf{2 5 7})\end{array}$ & $\begin{array}{c}\text { Não realiza } \\
(\mathbf{n}=\mathbf{5 8})\end{array}$ & p-valor \\
\hline Sexarca & & & & \\
\hline Antes dos 18 anos & $213(70,3)$ & $182(73,1) \dagger$ & $31(57,4)^{*}$ & $0,038^{1}$ \\
Após os 18 anos & $90(29,7)$ & $67(26,9)^{*}$ & $23(42,6) \dagger$ & \\
\hline Atividade sexual (semanal) & & & & \\
\hline Nenhuma & $89(28,3)$ & $65(25,3)^{*}$ & $24(41,4) \dagger$ & \\
Uma & $34(10,8)$ & $29(11,3)$ & $5(8,6)$ & $0,019^{1}$ \\
Duas & $49(15,6)$ & $36(14)$ & $13(22,4)$ & \\
Três & $54(17,1)$ & $48(18,7)$ & $6(10,3)$ & \\
Mais de três & $89(28,3)$ & $79(30,7) \dagger$ & $10(17,2)^{*}$ & \\
\hline Usa método contraceptivo? & & & & \\
\hline Não & $184(58,4)$ & $139(54,1)^{*}$ & $45(77,6) \dagger$ & $0,001^{1}$ \\
Sim & $131(41,6)$ & $118(45,9) \dagger$ & $13(22,4)^{*}$ & \\
\hline
\end{tabular}

Legenda: As variáveis categóricas são representadas como $\mathrm{n}$ (\%). ${ }^{1}$ : Teste G. *: A frequência observada foi inferior ao que seria esperado ao acaso. $\dagger$ : A frequência observada foi superior ao que seria esperado ao acaso.

Fonte: Fernandes LHCL, et al., 2020.

A Tabela 3 mostra que o fato das usuárias fumarem ou ingerirem bebida alcoólica não influencia a decisão de realizar ou não os exames preventivos.

Tabela 3 - Fatores de risco das usuárias do serviço de prevenção do câncer de colo uterino da Unidade Básica de Saúde escolhida, avaliadas no período de outubro de 2018 a janeiro de 2019, em uma cidade do Pará.

\begin{tabular}{lcccc}
\hline Variável & Geral & $\begin{array}{c}\text { Realiza } \\
(\mathbf{n = 2 5 7})\end{array}$ & $\begin{array}{c}\text { Não Realiza } \\
(\mathbf{n}=58)\end{array}$ & p-valor \\
\hline Fuma? & & & & \\
\hline Não & $293(93)$ & $239(93)$ & $54(93,1)$ & $0,800^{1}$ \\
Sim & $22(7)$ & $18(7)$ & $4(6,9)$ & \\
\hline Consome álcool? & & & & 0,9941 \\
\hline Não & $247(78,4)$ & $202(78,6)$ & $45(77,6)$ & \\
Sim & $68(21,6)$ & $55(21,4)$ & $13(22,4)$ &
\end{tabular}

Legenda: As variáveis categóricas são representadas como n (\%). 1: Teste G.

Fonte: Fernandes LHCL, et al., 2020.

Quanto a variáveis ligadas à qualidade de vida, observou-se que nem a prática ou não de atividade física, nem a frequência semanal de exercícios, nem o consumo frequente de frituras, nem o consumo frequente de frutas, verduras e legumes se associaram significativamente, de forma direta, à decisão de realizar ou não regularmente o exame (Tabela 4). 
Tabela 4 - Atividade física e alimentação das usuárias do serviço de prevenção do câncer de colo uterino da Unidade Básica de Saúde escolhida, avaliadas no período de outubro de 2018 a janeiro de 2019, em uma cidade do Pará.

\begin{tabular}{|c|c|c|c|c|}
\hline Variável & Geral & $\begin{array}{l}\text { Realiza } \\
(\mathrm{n}=257)\end{array}$ & $\begin{array}{c}\text { Não Realiza } \\
(n=58)\end{array}$ & p-valor \\
\hline \multicolumn{5}{|c|}{ Pratica atividade física? } \\
\hline Sim & $222(70,5)$ & $181(70,4)$ & $41(70,7)$ & \multirow{2}{*}{$0,905^{1}$} \\
\hline Não & $93(29,5)$ & $76(29,6)$ & $17(29,3)$ & \\
\hline \multicolumn{5}{|c|}{ Se pratica atividade física, quantas vezes por semana? } \\
\hline Até 2 & $25(29,4)$ & $20(27,8)$ & $5(38,5)$ & \multirow{2}{*}{$0,659^{1}$} \\
\hline Três ou mais & $60(70,6)$ & $52(72,2)$ & $8(61,5)$ & \\
\hline \multicolumn{5}{|c|}{ Tipo de alimentos mais consumidos? Fritura } \\
\hline Não & $160(50,8)$ & $135(52,5)$ & $25(43,1)$ & \multirow{2}{*}{$0,249^{1}$} \\
\hline Sim & $155(49,2)$ & $122(47,5)$ & $33(56,9)$ & \\
\hline \multicolumn{5}{|c|}{ Tipo de alimentos mais consumidos? Frutas, legumes, verduras } \\
\hline Não & $51(16,2)$ & $36(14)$ & $15(25,9)$ & \multirow[b]{2}{*}{$0,054^{1}$} \\
\hline Sim & $264(83,8)$ & $221(86)$ & $43(74,1)$ & \\
\hline
\end{tabular}

Legenda: As variáveis categóricas são representadas como $\mathrm{n}(\%) .{ }^{1}$ : Teste $\mathrm{G}$.

Fonte: Fernandes LHCL, et al., 2020.

A Tabela 5 mostra que não há associação entre o número de abortos e a decisão de realizar os exames, enquanto quem teve três ou mais partos é mais propenso a realizar os exames, quem teve três ou mais gestações idem, e quem realizou histerectomia também é mais propensa a realizar os exames.

Tabela 5 - Histórico ginecológico e obstétrico das usuárias do serviço de prevenção do câncer de colo uterino da Unidade Básica de Saúde escolhida, avaliadas no período de outubro de 2018 a janeiro de 2019, em uma cidade do Pará.

\begin{tabular}{|c|c|c|c|c|}
\hline Variável & Geral & $\begin{array}{l}\text { Realiza } \\
(n=257)\end{array}$ & $\begin{array}{l}\text { Não Realiza } \\
(n=58)\end{array}$ & p-valor \\
\hline \multicolumn{5}{|c|}{ Número de partos } \\
\hline Nenhum & $63(20,9)$ & $51(20,7)$ & $12(21,4)$ & \multirow{4}{*}{$0,008^{1}$} \\
\hline Um & $73(24,2)$ & $66(26,8) \dagger$ & $7(12,5)^{*}$ & \\
\hline Dois & $96(31,8)$ & $81(32,9)$ & $15(26,8)$ & \\
\hline Três ou mais & $70(23,2)$ & $48(19,5)^{*}$ & $22(39,3) \dagger$ & \\
\hline \multicolumn{5}{|c|}{ Número de gestações } \\
\hline Nenhuma & $61(19,7)$ & $49(19,4)$ & $12(21,1)$ & \multirow{4}{*}{$0,004^{1}$} \\
\hline Uma & $75(24,2)$ & $69(27,3) \dagger$ & $6(10,5)^{*}$ & \\
\hline Duas & $98(31,6)$ & $82(32,4)$ & $16(28,1)$ & \\
\hline Três ou mais & $76(24,5)$ & $53(20,9)^{*}$ & $23(40,4) \dagger$ & \\
\hline \multicolumn{5}{|c|}{ Número de abortos } \\
\hline Nenhum & $254(87,3)$ & 207 (87) & $47(88,7)$ & \multirow{2}{*}{$0,913^{1}$} \\
\hline Um ou mais & $37(12,7)$ & $31(13)$ & $6(11,3)$ & \\
\hline \multicolumn{5}{|c|}{ Realizou histerectomia? } \\
\hline Nenhuma & $238(75,6)$ & $203(79) \dagger$ & $35(60,3)^{*}$ & \multirow{3}{*}{$0,013^{1}$} \\
\hline Total & $21(6,7)$ & $16(6,2)$ & $5(8,6)$ & \\
\hline Parcial & $56(17,8)$ & $38(14,8)^{*}$ & $18(31) \dagger$ & \\
\hline
\end{tabular}

Legenda: As variáveis categóricas são representadas como $n(\%) .{ }^{1}$ : Teste G. *: A frequência observada foi inferior ao que seria esperado ao acaso. $\dagger$ : A frequência observada foi superior ao que seria esperado ao acaso. Fonte: Fernandes LHCL, et al., 2020.

A Tabela 6 mostra que não há relação de causa e efeito, em termos estatísticos, entre o histórico de doenças em si ou em familiares e a decisão de realizar ou não os exames periodicamente. 
Tabela 6 - Histórico de doenças das usuárias do serviço de prevenção do câncer de colo uterino da Unidade Básica de Saúde escolhida, avaliadas no período de outubro de 2018 a janeiro de 2019, em uma cidade do Pará.

\begin{tabular}{lcccc}
\hline Variável & Geral & $\begin{array}{c}\text { Realiza } \\
(\mathbf{n = 2 5 7 )}\end{array}$ & $\begin{array}{c}\text { Não Realiza } \\
(\mathbf{n = 5 8 )}\end{array}$ & p-valor \\
\hline Possui alguma doença crônica? & & & & \\
\hline Não & $231(73,3)$ & $190(73,9)$ & $41(70,7)$ & \multirow{2}{*}{$0,735^{1}$} \\
Sim & $84(26,7)$ & $67(26,1)$ & $17(29,3)$ & \\
\hline \multicolumn{1}{l}{ Há histórico de doenças crônicas na família? } & & & \\
\hline Não & $191(60,6)$ & $155(60,3)$ & $36(62,1)$ & \multirow{2}{*}{$0,921^{1}$} \\
Sim & $124(39,4)$ & $102(39,7)$ & $22(37,9)$ & \\
\hline Há histórico de câncer na família? & & & & \multirow{2}{*}{$0,333^{1}$} \\
\hline Não & $241(76,5)$ & $200(77,8)$ & $41(70,7)$ & $17(29,3)$ \\
Sim & $74(23,5)$ & $57(22,2)$ &
\end{tabular}

Legenda: As variáveis categóricas são representadas como $\mathrm{n}(\%) .{ }^{1}$ : Teste $\mathrm{G}$.

Fonte: Fernandes LHCL, et al., 2020.

Quanto aos conhecimentos sobre o exame e o histórico de atendimentos, observou-se que quem não sabe o que é o exame preventivo é mais propenso a não realizar o exame (mostrando a importância de campanhas de esclarecimento, por exemplo). Também, quem não conhece os horários de atendimento é mais propenso a não realizar o exame, assim como quem realizou os exames em anos recentes (2018 e 2019) é mais propenso a manter a periodicidade.

Além disso, os resultados detalham os meios de comunicação reportados pelas usuárias. O veículo mais reportado foi a TV, com 264 mulheres relatando ter acesso a ela. Observa-se que não houve associação entre o meio de comunicação acessível e a decisão de realizar ou não o exame.

A doença crônica mais relatada pelas usuárias foi a hipertensão arterial (com 61,4\% das usuárias relatando possuir hipertensão arterial). Porém, o tipo de doença crônica não se associou significativamente à decisão de realizar ou não o exame $(p=0,424)$.

O histórico de câncer na família associou-se significativamente à decisão de realizar ou não o exame, de forma que quem já teve câncer de colo do útero na família foi mais propenso a realizar os exames periodicamente. $O$ tipo de método contraceptivo utilizado também associou-se significativamente à decisão de realizar ou não o exame, sendo que as mulheres que realizam o controle por tabelinha são mais propensas a não realizar o exame.

A maioria das usuárias relatou que a utilidade do exame é a prevenção de câncer, porém, estas proporções não diferiram significativamente entre as mulheres que realizam ou que não realizam os exames periodicamente. Além disso, detalha todos os motivos relatados como impeditivos da realização periódica dos exames. Observa-se que a falta de tempo foi citada por mais da metade das usuárias, enquanto a falta de transporte e a falta de informações sobre o exame foram os motivos menos relatado.

\section{DISCUSSÃO}

O exame de Prevenção de Câncer de Colo Uterino (PCCU) possui extrema importância na saúde física e psicológica da mulher, possuindo diversas barreiras que são reflexos para não realização durante a vida. Dentre esses contextos, a literatura corrobora que há diminuição da taxa de adesão ao exame por mulheres que são viúvas por motivo da associação de necessidade da realização do exame apenas quando há atividade sexual.

Além disso, o baixo nível de escolaridade e sócio econômico são fatores que contribuem para a decisão e escolha de submeter-se a realização do exame, sendo uma medida inversamente proporcional pois a medida que diminui os fatores citados aumenta a prevalência de mulheres que não realizam o exame, comprometendo a qualidade de vida, aumentando as taxas de diagnóstico tardio de casos de câncer e ressaltando a educação em saúde da equipe de atenção básica como imprescindível na transformação dessa realidade (OLIVEIRA MM, 2007; CESARIN MR, 2011; SOUZA GG, 2011). 
A faixa etária média das mulheres que procuram esse serviço é de 41,8 anos sendo discordante com diversos estudos que relatam uma média mais precoce (entre 20-34 anos) de procura, contudo avaliando a análise das idades, todos os estudos analisados demonstram a diminuição da quantidade absoluta de mulheres acima de 55 anos que solicitam o exame influenciado pela possível associação de pré-requisito para realização do PCCU ser a atividade sexual (CONDE CR, et al., 2018; DUARTE DV, et al., 2016).

Outros fatos encontrados na pesquisa, que corroboram esse ideal de necessidade da associação da atividade sexual para não realização do PCCU, além de serem similares ao identificados em diversas pesquisas semelhantes em outras regiões do país são: perfil de mulheres que não utilizam métodos contraceptivos e quem relata não possuir atividade sexual reforçando esse contexto ao coletar que mulheres que possuem mais de três relações sexuais são mais propensas para a realização do exame (RODRIGUES DE, et al., 2016; NAVARRO C, et al., 2015). Além disso, ressalta-se em diversas pesquisas que a não realização do exame pelo público gestante fundamentado na ideia de possibilidade de prejudicar o binômio materno-fetal (TERLAN RJ, et al., 2018; ROSA ARR, et al., 2018).

A não realização no público gestante pode gerar o não diagnóstico de patologias sérias que podem prejudicar o binômio materno-fetal causando restrição de crescimento intrauterino, malformação fetal e até abortos espontâneos e partos pré-termos (TERLAN RJ, et al., 2018).

Tal fato mostra-se importante no manejo das pacientes pois para a realização do exame de PCCU é necessário fazer a inspeção da parede e colo uterino previamente, sendo identificado anormalidades que não são sugestivas de câncer de colo de útero e não dependem de atividade sexual para desenvolverem (exemplo vulvovaginites e atrofia sugestiva de menopausa) mas são patologias que diminuem a qualidade de vida, provocam dores físicas e, em algumas doenças crônicas, infertilidade (BARBOSA IR, 2017). Sendo assim, o exame de preventivo possui benefícios extras que não atingem a totalidade das mulheres.

Ressalta-se que os hábitos de vida (tabagismo, etilismo, atividade física, alimentação saudável) não se associaram estatisticamente com a realização ou não do exame preventivo concordando com a literatura vigente na área, contudo destaca-se um número expressivo de mulheres tabagistas, não realizam atividade física e que tem fritura como alimento principal durante o estudo da pesquisa sendo um perfil de paciente que precisa ser combatido para prevenir diversas doenças metabólicas e crônicas como hipertensão arterial sistêmica (inclusive já encontrado em $61,4 \%$ das pacientes pesquisadas), diabetes mellitus (também encontrado em quase $1 / 4$ das pacientes coletadas), infarto agudo do miocárdio, obesidade, dislipidemia e alguns tipos de câncer como neoplasia de mama, ressaltando a atenção básica como ambiente propício para prevenção, educação em saúde e diagnóstico de diversas patologias mesmo em mulheres que não realizam o exame de preventivo (CAMPOS EA, et al., 2017).

A relação de causa e efeito entre histórico familiar de doenças gerais (como hipertensão arterial sistêmica) e a decisão de realizar ou não os exames periodicamente não foi encontrada no estudo contrapondo a associação, evidenciada no mesmo estudo, entre casos de câncer na família e a escolha de realizar o exame, sendo esse fato corroborado na literatura onde diversos estudos apontam o mesmo contexto explicando que o medo de ter a doença é maior em quem conviveu com familiar que possuiu quaisquer tipos de câncer, sobrepondo o ideal de atividade sexual como pré-requisito para a realização do exame de PCCU e aumentando a procura por mudanças em estilo de vida, controle de doenças descompensadas e rastreio de diversas patologias (DISCACCIATI MG, et al., 2014)

Além do aspecto de qualidade de vida, existem diversas barreiras que impedem a não procura da paciente em realizar o exame de preventivo. Dentre elas destaca-se a falta de conhecimento como um fator que predominou e encontrou associação estatística com a não realização do PCCU, corroborando com aspectos de diversas pesquisas em várias regiões do país que mostram a importância de campanhas de educação em saúde promovidas pela atenção básica, conhecimento de profissionais de saúde nessa área a fim de alertar a paciente que vai à unidade básica de saúde por outro motivo que não o exame de preventivo e a busca ativa de assistentes comunitários de saúde (ACS's) e profissionais de saúde em identificar e orientar as mulheres durante as visitas domiciliares a fim de diminuir o desconhecimento sobre os objetivos e motivos do exame (RODRIGUEZ G, et al., 2015). 
Além desse fato, destacam-se não conhecer os horários de atendimento da unidade de saúde, falta de tempo para ir realizar o exame por motivos relatados como período discordante com horários da unidade saúde, medo ou vergonha de realizar o exame, não encontrar importância para realização e não foi bem tratada quando foi a unidade de saúde anteriormente como outros fatores que contribuem para a não realização do exame corroborando com a literatura atual nesses aspectos, sendo causado por diversos motivos como períodos inflexíveis de funcionamento de unidades básicas de saúde, falta de campanhas em educação em saúde envolvendo toda a equipe do local a fim de apresentar a importância da realização do preventivo bem como estreitar a relação entre profissionais da atenção básica e público do exame (CONDE CR, et al., 2018; DUARTE DV, et al., 2017).

\section{CONCLUSÃO}

Logo, esta pesquisa foi importante para reconhecer o perfil socioeconômico de mulheres e motivações para não realização do exame de PCCU na área pesquisa, sendo uma pesquisa com metodologia que deve ser difundida para ser realizada em outras áreas do país a fim de focar as campanhas de educação em saúde nos temas que são barreiras e melhorar a qualidade de vida das pacientes que não realizam o exame, convencendo e informando sobre os objetivos, fundamentações e benefícios para realizar o exame de preventivo. Dessa forma, são necessárias mais pesquisas semelhantes a atual com intuito de criar referencial bibliográfico suficiente a fim de desenvolver medidas, protocolos, materiais para amenizar esta problemática bem como o desenvolvimento de infraestrutura e logística para o atendimento dessas pacientes nas várias esferas e eixos (primário, secundário, terciário e quaternário) do Sistema Único de Saúde (SUS) para o melhor atendimento e a melhoria da qualidade de vida biopsicossocial das pacientes com os diversos resultados do exame de colpocitologia oncótica (PCCU).

\section{REFERÊNCIAS}

1. SILVA MRF, et al. Continuidade assistencial a mulheres com câncer de colo de útero em redes de atenção à saúde: estuo de caso, Pernambuco. Rev Saúde Debate, 2016, 40(110):107-119.

2. BARCELOS MRB, et al. Qualidade do rastreamento do câncer de colo uterino no Brasil: avaliação externa do PMAQ. Rev Saúde Pública, 2014, 48(2):240-248.

3. MELO MCS, et al. O enfermeiro na prevenção do câncer do colo do útero: o cotidiano da atenção primária. Revista Brasileira de Cancerologia; 2012, 58(3):389-398.

4. SOUZA AF, et al. Conhecimento de mulheres sobre HPV e câncer do colo do útero após consulta de enfermagem. Revista Brasileira de Cancerologia, 2015; 61(4):343-350

5. AMARAL MS. Prevenção do câncer de colo de útero: a atuação do profissional enfermeiro nas Unidades Básicas de Saúde. Revista Científica FacMais, 2017, 8(1).

6. FACCHINI LA, et al. Governance and health system performance: national and municipal challenges to the Brazilian Family Health Strategy. In: REICH, M. R; TAKEMI, K. (Editors). Governing health systems: for nations and communities around the world. Brookline: Lamprey \& Lee, 2015.

7. DAMACENA AM, et al. Rastreamento do câncer do colo do útero em Teresina, Piauí: estudo avaliativo dos dados do Sistema de Informação do Câncer do Colo do Útero, 2006-2013. Rev Epidemiologia e Serviços de Saúde, 2017, 26(1):71-80

8. CESARIN MR. Educação em saúde para prevenção do câncer de colo do útero em mulheres do município de Santo Ângelo/RS. Ciência e saúde coletiva, 2011; 16(9):3925-3932.

9. SOUZA GG. A importância de ações educativas para prevenção do câncer de colo uterino no contexto da Estratégia Saúde da Família. Monografia. (Especialização em Atenção Básica à Saúde da Família) - Universidade Federal de Minas Gerais, Teófilo Otoni, 2011

10. OLIVEIRA MM. Percepção das usuárias sobre as ações de prevenção do câncer do colo do útero na Estratégia Saúde da Família em uma Distrital de Saúde do município de Ribeirão Preto, São Paulo, Brasil. Rev. Bras. Saúde Matern. Infant., 2007; 7(1):31-38.

11. CONDE CR, et al. Características sociodemográficas, individuales y de programación de las mujeres con cáncer cervical. Enferm. glob., 2018, 17(49): 348-380.

12. DUARTE DV, et al. Prevalence of Human Papillomavirus Infection and Cervical Cancer Screening among Riverside Women of the Brazilian Amazon. Rev. Bras. Ginecol. Obstet, 2017, 39(7):360-357. 
13. RODRIGUES DE, et al. Barriers to prevention of cervical cancer in the city of Porto Velho Velho, Rondônia, Brazil. Invest. educ. enferm, 2016, 34(1): 59-67.

14. NAVARRO C, et al. Cervical cancer screening coverage in a high-incidence region. Rev. Saúde Pública, 2015, 49(17).

15. TERLAN RJ, et al. Não realização de citopatológico de colo uterino entre gestantes no extremo sul do Brasil: prevalência e fatores associados. Ciênc. Saúde coletiva, 2018, 23(11): 3557-3566.

16. ROSA ARR, et al. Exame citopatológico do colo do útero: investigação sobre o conhecimento, atitude e prática de gestante. Cogitare enferm., 2018, 23(2): e52589.

17. BARBOSA IR. Regional and Socioeconomic Differences in the Coverage of the Papanicolau Test in Brazil: Data from the Brazilian Health Survey 2013. Rev. Bras. Ginecol. Obstet, 2017, 39(9):480-487.

18. CAMPOS EA, et al. "Uma doença da mulher": experiência e significado do câncer cervical para mulheres que realizaram o Papanicolau. Interface, Botucatu, 2017, 21(61):385-396

19. DISCACCIATI MG, et al. Por que a prevalência de resultados citopatológicos do rastreamento do câncer do colo do útero pode variar significativamente entre duas regiões do Brasil? Rev. Bras. Ginecol. Obstet, 2014, 36(5):192-197

20. RODRIGUEZ G, et al. Conocimientos, actitudes y prácticas sobre el test de Papanicolaou y estadificación del cáncer de cuello uterino. Rev. Méd. Urug, 2015, 31(4):231-240. 